\title{
Trend of Influenza Like Illness in Shiro Meda Health Center Sentinel Site, 2013 to 2017, Addis Ababa, Ethiopia, 2018
}

\author{
Habtamu Tilahun Guadie ${ }^{1,{ }^{*}, \text { Zewdu Assefa Edea }}{ }^{1}$, Girma Taye ${ }^{2}$, Adamu Tayachew Mekonen ${ }^{1}$ \\ ${ }^{1}$ Public Health Emergency Management Center, Ethiopian Public Health Institute, Addis Ababa, Ethiopia \\ ${ }^{2}$ Field Epidemiology Training Programme, Addis Ababa University, Addis Ababa, Ethiopia
}

Email address:

Habtamutilahun5@gmail.com (H. T. Guadie), zedhiwot05@gmail.com (Z. A. Edea), girmataye2009@gmail.com (G. Taye)

${ }^{*}$ Corresponding author

\section{To cite this article:}

Habtamu Tilahun Guadie, Zewdu Assefa Edea, Girma Taye, Adamu Tayachew Mekonen. Trend of Influenza Like Illness in Shiro Meda Health Center Sentinel Site, 2013 to 2017, Addis Ababa, Ethiopia, 2018. World Journal of Public Health. Vol. 6, No. 1, 2021, pp. $13-16$. doi: 10.11648/j.wjph.20210601.13

Received: September 2, 2020; Accepted: December 11, 2020; Published: March 30, 2021

\begin{abstract}
Introduction: Influenza is a respiratory illness caused by influenza virus that can spread very easily from person to person. The virus is spread through the air by the exchange of fluid droplets from the mouth or nose of one person to another person due to sneezing and coughing. The manifestations of illness (fever, cough, sore throat) caused by the influenza virus are usually mild to moderate but for some it could be severe, leading to hospitalization and even death. In Ethiopia, influenza sentinel surveillance has been launched in 2008 with one ILI sentinel site (Shiromeda Health Center) and one SARI sentinel site (Yekatit 12 Hosp). Ongoing analysis of surveillance data is important for detecting outbreaks and unexpected increases or decreases in disease occurrence, monitoring disease trends, and evaluating the effectiveness of disease control programs and policies. This trend analysis was conducted to characterize ILI cases in person, place and time, determine the positivity rate of the sentinel site and put possible recommendations based on the findings. Methods: We reviewed five years Influenza (2013 to 2017) data of Shiro meda health center. Data was cleaned prior to analysis. Descriptive analysis by person, place and time was done using Microsoft excel 2013. Results displayed using narration, graphs and tables. Results: A total of 1,489 cases of Influenza like illness reported from Shiro meda health center for the last five years, of which $950(64 \% 0$ were females and the remaining $539(46 \%)$ were males. Out of the total reported cases, $98 \%$ were from Gullele sub-city. The most affected age group was $15-44(49 \%)$ followed by 5-14 (32\%). The positivity rate of the site was $30.31 \%$. Of those positive cases for Influenza, 20\% were Influenza type A and the remaining were Influenza type B. Some important characteristics of patients are missing. Conclusion: Almost all of the cases were reported from Gullele sub-city. The most affected age group was 15-44 followed by 5-14. Influenza type A and B are circulating in the sentinnel site. Regular data analysis and supevision is required to improve the quality of data produced in the sentinnel site.
\end{abstract}

Keywords: Influenza, Senitinnel Site, Analysis, Shiro Meda Health Center, Ethiopia

\section{Introduction}

Influenza is a respiratory illness caused by influenza virus that can spread very easily from person to person. The virus is spread through the air by the exchange of fluid droplets from the mouth or nose of one person to another person due to sneezing and coughing. The manifestations of illness (fever, cough, sore throat) caused by the influenza virus are usually mild to moderate but for some it could be severe, leading to hospitalization and even death. The influenza virus circulates around the world and undergoes continuous evolution by antigenic drift which causes annual epidemics. In rare instances the virus may change completely called 'antigenic shift', and result in the emergence of novel influenza viruses [1].

There are 3 types of seasonal influenza viruses, types A, B, and C. Influenza type A viruses are further classified into subtypes according to the combinations of 2 different 
proteins, the haemagglutinin $(\mathrm{H})$ and the neuraminidase $(\mathrm{N})$, located on the surface of the virus. The subtypes of influenza A viruses currently circulating among humans are influenza A (H1N1) and A (H3N2) subtypes. The circulating influenza $\mathrm{A}(\mathrm{H} 1 \mathrm{~N} 1)$ is also written as $\mathrm{A}(\mathrm{H} 1 \mathrm{~N} 1)$ pdm09 as it caused the pandemic in 2009 and subsequently replaced the seasonal influenza A (H1N1) virus which had circulated prior to 2009. Only influenza type A viruses are known to have caused pandemics. Circulating influenza $B$ viruses can be divided into 2 main groups (lineages), referred to as B/Yamagata and $\mathrm{B} /$ Victoria lineages. Influenza B viruses are not classified into subtypes [1].

In Ethiopia, influenza sentinel surveillance has been launched in 2008 with one ILI sentinel site (Shiromeda Health Center) and one SARI sentinel site (Yekatit 12 Hospital). Furthermore, additional two ILI sentinel sites (Akaki Kality and Kolfe health Centers) are added in 2010. Subsequently, in 2013 SARI sentinel sites were established in four mega regional state capitals. Currently there are three ILI and Five SARI sentinel sites providing both weekly aggregated and throat swab samples. Some baseline data has been generated in all eight sentinel sites, namely Shiromeda Health Center, Yekatit 12 Hospital, Kolfe Health Center, Akaki Health Center, Adama Hospital, Adare Hospital, Felegehiwot Hosapital and Mekelle Hospital. Ongoing analysis of surveillance data is important for detecting outbreaks and unexpected increases or decreases in disease occurrence, monitoring disease trends, and evaluating the effectiveness of disease control programs and policies. The study was conducted to characterize ILI patients in terms of place person and time, the trend of cases over time and determine the positivity rate of ILI cases in shiro meda health center.

\section{Methods and Materials}

Five years data (2013-2017) of Shiro meda health center was received and compiled from Public Health Emergency Management center of Ethiopian Public Health Institute. The data was cleaned prior to analysis. After cleaning, the data was analyzed using Micro soft excel 2013. Attack rates per year were calculated using number of cases reported per year divided by total population for the respective year multiplied by 100,000 . Tables and graphs were used to display results. The analysis was conducted from $1^{\text {st }}$ to $15^{\text {th }}$ February 2018.

\section{Case Definitions}

\subsection{Influenza Like Illness (ILI)}

A person, child or adult with sudden onset of fever $>38^{\circ} \mathrm{C}$ and Cough or sore throat in the absence of other diagnoses.

\subsection{Severe Acute Respiratory Infection (SARI)}

Any severely ill person presenting with manifestations of acute lower respiratory infection with history of fever or measured fever $\left(>=38^{\circ} \mathrm{C}\right)$ and cough or sore throat and shortness of breath, or difficulty of breathing with or without Clinical or radiographic findings of pneumonia or any person who died of an unexplained respiratory illness.

\section{Results}

A total of 1,486 ILI cases reported from Shiro Meda health center from 2013 to 2017. All of them were reported from Addis Ababa city. Out of all reported cases, 1,468 (98\%) cases were from Gullele sub-city.

Out of the total reported cases for the last five years, 950 $(64 \%)$ were females and the remaining 539 (46\%) were males.

Age group 15-44 comprises $49.69 \%$ of the total reported cases followed by $5-14$ age group which is $32.43 \%$.

The trend of cases over the last five years looks decreasing. The figure below shows the incidence rate of ILI cases in Addis Ababa city for the last five years per year per 100,000 populations.

Out of the total reported cases in national Influenza laboratory, $448(30.31 \%)$ cases tested positive for Influenza virus. The following table shows the positivity status of reported cases in Shiro meda Health center from year 2013 to 2017.

Table 1. Positivity status of reported ILI cases from Shiro meda health center in Addis Ababa city from year 2013 to 2017, February 2018.

\begin{tabular}{lll}
\hline Status & Number of cases & Percentage \\
\hline Positive & 448 & $30.31 \%$ \\
Negative & 1022 & $69.14 \%$ \\
Intermediate & 7 & $.55 \%$ \\
Total & 1478 & $100 \%$ \\
\hline
\end{tabular}

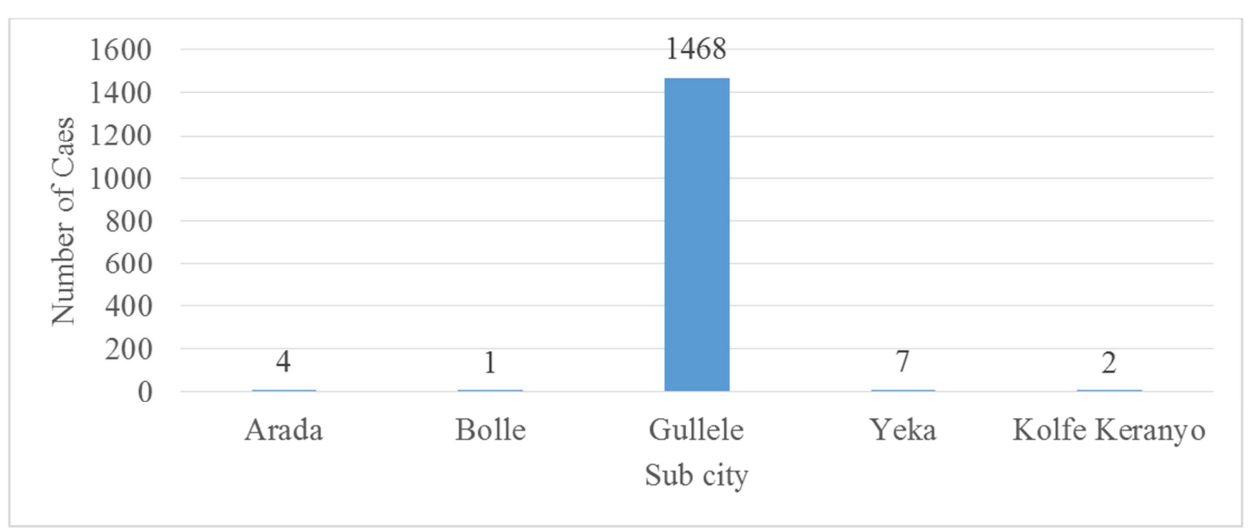

Figure 1. Influenza like illness cases in Shiro meda health center by sub-city from year 2013 to 2017, February 2018. 


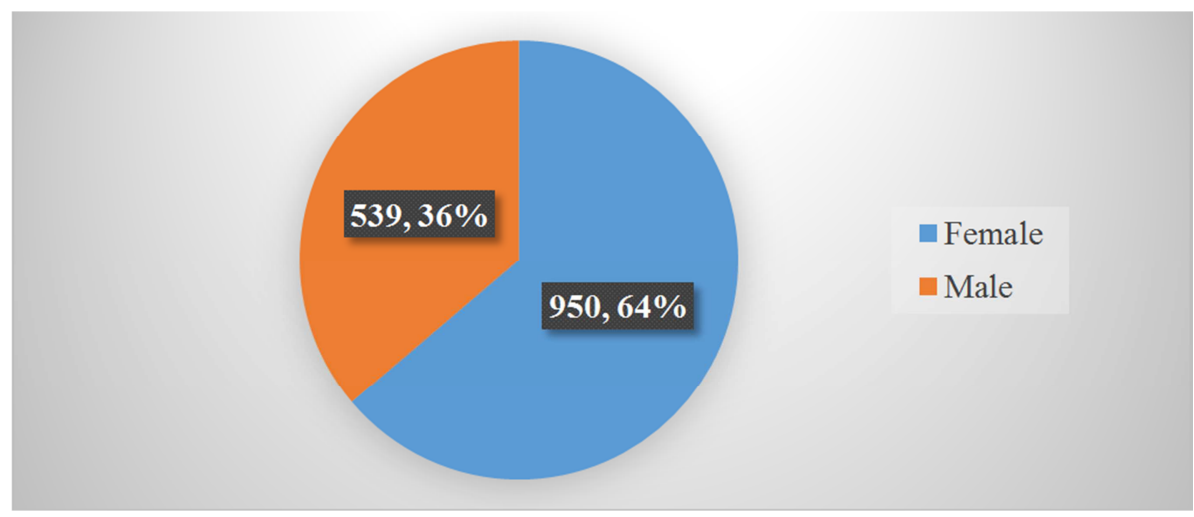

Figure 2. ILI cases Shiro meda Health center by sex year from 2013 to 2017, February 2018.

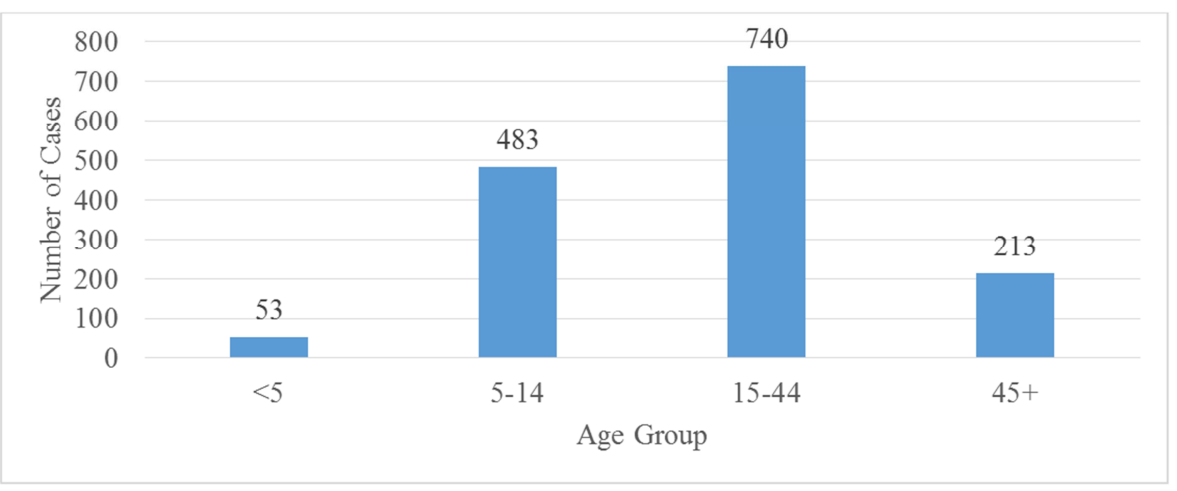

Figure 3. ILI cases of Shiro meda Health center by Age group year 2013 to 2017, February 2018.

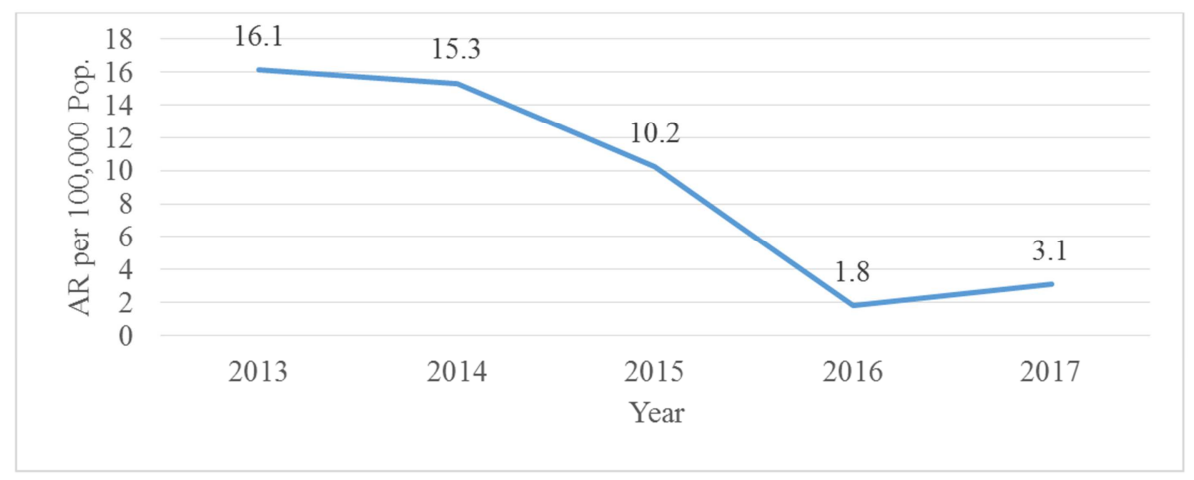

Figure 4. Attack rate of ILI in Addis Ababa city per year, per 100,000 in Shiro meda HC, 2013 to 2017, February 2018.

\section{Discussions}

Most of the cases were reported from Gullele sub city hence the sentinel site is found under this sub city and it is also similar with another study done in Ethiopia in 2012 of which around $70 \%$ of cases were from this sub city [2]. This study revealed that the Age Group 15-44 is mostly affected compared to others which is in contrast with a study done in Ethiopia in 2012 which revealed that the most affected Age Group is 0-4 [2]. In a study done in Kenya in $201280 \%$ of the cases were in the Age Group of 0-4 which is also in contrast to this study. In this study a single site is used and the study in Kenya covered all over the country, so this might be the reason for discrepancies in the results [3]. Family studies conducted in Houston and Seattle also demonstrated that high rates of infection in school-age children and the importance of school children as vehicles of infection within families [6,8]. Additionally the study conducted in Peru on influenza from 2006-2008 revealed that the prevalence of influenza $\mathrm{A}$ and $\mathrm{B}$ viruses were significantly higher in ILI patients older than 5 years of age [7]. This data analysis uncovered that through years 2013 to 2017 Influenza A and B are circulating in Shiro Meda Health Center Influenza Sentinel site. Compared to Influenza $\mathrm{B}$, Influenza $\mathrm{A}$ is predominantly circulating in this Health Center (64.4\% versus. 33.6\%), which is not consistent with a study done in Ethiopia between 2008 and 2011 which was $86.6 \%$ versus $13.4 \%$ [2]. This might be due to, in this study cases from a single sentinel site (Shiro Meda HC) was enrolled and the study between 2008 and 2012 was done on sites all over the city. Similarly influenza $A$ is mainly circulating in Kenya than influenza B $(69 \%$ 
versus $31 \%$ ) in a research done in 2012 [3]. This finding is also comparable with a study conducted between 2009 and 215 in Ethiopia [10]. In another study done in South Africa from year 2009-2012, of those Tested positive cases for Influenza, 37\% were Influenza A [5]. Study conducted in Thailand in 2001 also shows that influenza $\mathrm{A}$ is by and large occurred than influenza B (69.8\% versus 30.2\%) [3]. This result is also comparable with the research done in West Africa between 2010 and 2012 which showed that, out of the total tested cases $62.8 \%$ were Influenza $\mathrm{A}$ and the remaining $37.2 \%$ were positive for Influenza B [9].

\section{Conclusions}

Based on this study finding Influenza A (64.4\%) is the most circulating type the site and from Influenza A subgroups seasonal Influenza $(\mathrm{A} / \mathrm{H} 3)$ is the dominant one. Gullele subcity $(98.58 \%)$ is the most affected sub-city. The Age Group $15-44(49.69 \%)$ is the mostly affected Age Group in the site. The site misses some important variables of a patient, making the data under expected quality. Continuous feedback and supervision should be conducted regularly to improve the quality of data produced by the site. Documentation of important characteristics of patients should be improved.

What is known?

1) It is a respiratory diseases caused by virus

2) It caused by three strains of influenza viruses (A, B and C)

3) It is known to epidemics and pandemics in the world

What this study adds?

1) The Influenza strains circulating in the sentinel site

2) The trends of Influenza like illness in this sentinel site for the last five years

3) The most predominant strain of influenza virus in the site and the positivity rate of the site out of the total collected samples

\section{Abbreviations}

AR-Attack Rate

ILI-Influenza like Illness

HC-Health Center

SARI-Severe Acute Respiratory Illness

\section{Competing Interests}

All authors don't have any possible conflicts of interest.

\section{Authors' Contribution}

HT proposed and designed the analysis, carried out data cleaning, categorizing, analysis and write-up of the manuscript. All authors have participated in the interpretation of findings and review of the manuscript.

\section{Acknowledgements}

The authors wish to thank Ethiopian Public Health Institute, Public Health Emergency Management Center for providing five years data of Influenza and Addis Ababa University School of Public Health, Field Epidemiology Program for letting us undergo this study.

\section{References}

[1] Ministry of Health Bhutan, second edition, 2014, Operational definition for Influenza like Illness and Severe acute Respiratory Infection.

[2] Influenza like Illness Sentinel Surveillance-Ethiopia 20082011 Abyot Bekele1, 3, J. Daddi1, A. Worknesh2, Z. Etshiwot1, M. Mesfin2, D. Gelila2, K. Wubayehu2 Ethiopian Health and Nutrition Research Institute-Public Health Emergency Management Center, 2Ethiopian Health and Nutrition Research Institute- National Influenza Laboratory.

[3] Philip M. 2012, Influenza Epidemiology in Kenya.

[4] Pranee T. 2001, Isolation and Identification of Influenza Virus Strains Circulating in Thailand.

[5] Epidemiology of Influenza Virus Types and Subtypes in South Africa, 2009-2012.

[6] Glezen WP, Couch RB, 1978. Inter pandemic influenza in the Houston area.

[7] V. Alberto Laguna-Torres; 2006-2008, Influenza-Like Illness Sentinel Surveillance in Peru.

[8] Taber LH, Paredes A, Glezen WP, et al. 1981, Infection with influenza A/Victoria virus in Houston families.

[9] Epidemiology of influenza in West Africa after the 2009 influenza A (H1N1) pandemic, 2010-201.

[10] Woyessa, A. B., Mengesha, M., Belay, D. et al. Epidemiology of influenza in Ethiopia: findings from influenza sentinel surveillance and respiratory infection outbreak investigations, 2009-2015. BMC Infect Dis 18, 449 (2018). https://doi.org/10.1186/s12879-018-3365-5. 